\title{
Laparoscopic distal pancreatosplenectomy for isolated blunt traumatic pancreatic laceration: A case report and review of current literature
}

\author{
Ken Min Chin ${ }^{1}$, Ye-Xin Koh ${ }^{1}$, and Brian K. P. Goh ${ }^{1,2}$ \\ ${ }^{1}$ Department of Hepatopancreatobiliary and Transplant Surgery, Singapore General Hospital, \\ ${ }^{2}$ Duke-National University of Singapore (NUS) Medical School, Singapore
}

\begin{abstract}
Pancreatic injuries are often associated with trauma and occur most commonly in combination with other solid organ injuries. Management strategies for pancreatic injuries include conservative, endoscopic, percutaneous and surgical intervention. Literature on the laparoscopic approach to management of pancreatic trauma is rare and poorly reviewed. We describe a case report of successful and uncomplicated laparoscopic distal pancreatosplenectomy (LDP) for a patient suffering from isolated traumatic pancreatic tail transection. A literature review was performed with regards to the indications for intervention and different modalities of treatment for traumatic pancreatic lacerations. A review and comparison was also made between the scarce pre-existing reports of the laparoscopic approach to pancreatic resection in the setting of trauma. The laparoscopic approach to pancreatic resection, in the setting of trauma, can be considered as a viable alternative to open surgery. Moving forward, further studies with larger patient numbers will be needed to compare the outcomes between the open and laparoscopic approach. (Ann Hepatobiliary Pancreat Surg 2019;23:408-413)
\end{abstract}

Key Words: Pancreas; Wounds and injuries; Laparoscopy; Pancreatectomy

\section{INTRODUCTION}

Pancreatic injuries are most commonly caused by blunt trauma and occur almost exclusively in a young population. ${ }^{1,2}$ The diagnosis of pancreatic injury is most commonly made on computer tomography (CT) of the abdomen and pelvis. Management strategies for pancreatic injuries include conservative observation, endoscopic stenting, angioembolization, percutaneous drainage of collections and lastly, surgery. Surgical intervention is most frequently indicated in patients when injuries to the pancreas involve the main pancreatic duct. ${ }^{2-5}$ There is a paucity of literature on the laparoscopic approach to pancreatic resection in the setting of trauma, largely owing to the rarity of both the condition as well as the treatment modality. We describe a case report of successful and uncomplicated laparoscopic distal pancreatosplenectomy (LDP) for a patient suffering from isolated traumatic pancreatic tail transection and review the current literature.

\section{CASE}

This case involves a 22-year-old female with no significant past medical history. She presented to the emergency department with a 2-day history of severe epigastric pain following a road traffic accident. The patient, a motorcycle pillion rider, was flung at high speed when the vehicle hit a curb. She was unable to recall any specific abdominal trauma. On examination, she was afebrile, hemodynamically stable, conscious and oriented with tenderness and guarding over the epigastrium extending to the left hypochondrium. There was no rebound tenderness or peritonism and there were no signs suggestive of retroperitoneal hemorrhage.

Her initial laboratory investigations were unremarkable except for raised amylase (592 U/L) and lipase (373 U/L).

Received: December 26, 2018; Revised: July 20, 2019; Accepted: July 25, 2019

Corresponding author: Brian K. P. Goh

Department of Hepatopancreatobiliary and Transplant Surgery, Singapore General Hospital, 20 College Road, 169856, Singapore Tel: +65-6321-4051, Fax: +65-6220-9323, E-mail: bsgkp@hotmail.com

Copyright (C) 2019 by The Korean Association of Hepato-Biliary-Pancreatic Surgery

This is an Open Access article distributed under the terms of the Creative Commons Attribution Non-Commercial License (http://creativecommons.org/ icenses/by-nc/4.0) which permits unrestricted non-commercial use, distribution, and reproduction in any medium, provided the original work is properly cited. Annals of Hepato-Biliary-Pancreatic Surgery • pISSN: 2508-5778 - eISSN: 2508-5859 
Liver panel, chest $\mathrm{x}$-ray and abdominal $\mathrm{x}$-ray were normal. In view of persistent and severe epigastric pain, a $\mathrm{CT}$ of the abdomen and pelvis was performed. This revealed a discrete linear wedge-shaped cleft of hypoattenuation at the junction of the body and tail of the pancreas in keeping with an $80 \%$ laceration through the pancreas at this location (Fig. 1). There was an associated complete transection of the main pancreatic duct. A hyperdense focus was identified within the wedge-shaped laceration representing a hematoma (Fig. 2). Apart from mild peritoneal enhancement suggesting mild early peritonitis, there was no evidence of other solid organ injuries and the rest of the pancreatic parenchyma was normal.

The patient underwent diagnostic laparoscopy and LDP on day 3 of the injury. A $12-\mathrm{mm}$ paraumbilical port was first placed for the laparoscope. Two laparoscopic 5-mm working ports were inserted into the epigastrium and right hypochondrium and one 12-mm working port in the left iliac fossa. A thorough diagnostic laparoscopy was performed. Intra-operatively, the examination of the peritoneal cavity was relatively unremarkable with no other solid organ damage. Two PDS 2-0 stitches were used to suture and tack the stomach up to the anterior abdominal wall. The gastrocolic ligament was divided and the lesser sac entered, revealing a large retroperitoneal and lesser sac hematoma surrounding the pancreas and extending into the transverse mesocolon, associated with significant pancreatitis. There was a $90 \%$ transection at the junction between the body and tail of the pancreas. The short gastric

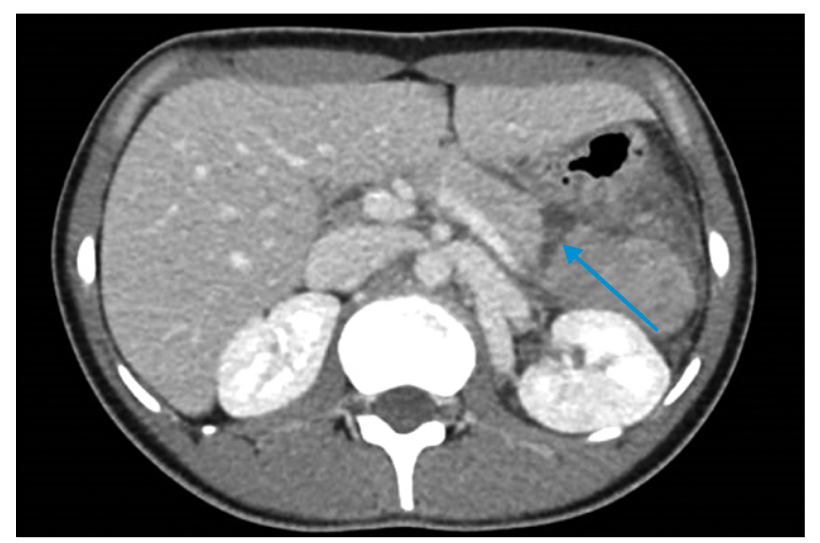

Fig. 1. CT abdomen pelvis showing a discrete linear wedgeshaped cleft of hypoattenuation at the junction of the body and tail of the pancreas in keeping with an $80 \%$ laceration through the pancreas, and an associated complete transection of the main pancreatic duct. vessels were divided, splenic flexure mobilized and splenic vessels identified. Retroperitoneal mobilization of the pancreas began at the inferior border of the pancreas, at the level of the site of transection. The pancreas was then slung to expose the region proximal to the level of transection. The splenic artery and vein were taken separately in between surgical clips. The pancreas was mobilized 2-cm proximal to the transection and an EndoGIA stapler (Covidien, Norwalk, Conn) was used to divide the pancreas. Tissue glue was applied and specimens were retrieved (spleen mosselated before retrieval) using an Endobag (Covidien). A single surgical drain was left in the splenic bed after copious wash and meticulous hemostasis. The total blood loss was $400 \mathrm{ml}$ and no transfusion was required. Total operative time was 122 minutes.

Post-operatively, the patient's recovery was uneventful. The surgical drain was removed after 3 days and she was discharged well 5 days after surgery.

\section{DISCUSSION}

The vast majority of pancreatic injuries are caused by blunt trauma $(>50 \%)$, with motor vehicle accidents being the most common etiology and accounting for up to half of these cases. ${ }^{1}$ Pancreatic injury is almost always associated with other intra-abdominal injuries, the most common being hepatobiliary injuries, duodenal injuries and injuries to major abdominal blood vessels. ${ }^{2-4}$ The pancreatic head is the most frequent site of injury, with damage to the body and tail occurring in a minority of cases. ${ }^{2}$ Classifi-

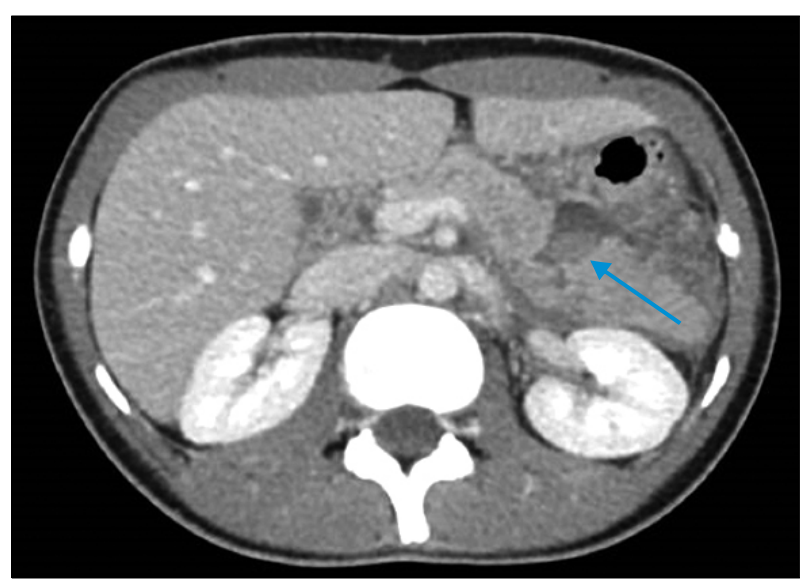

Fig. 2. CT abdomen pelvis showing a hyperdense focus within the wedge-shaped laceration representing a hematoma. 
cation of severity of pancreatic injury is according to the pancreatic injury scale described by the American Association for the Surgery of Trauma (AAST). ${ }^{2}$

Endoscopic evaluation is commonly undertaken only after a diagnosis of pancreatic injury is suspected on initial radiological investigations. The objective of endoscopic retrograde cholangiopancreatography (ERCP) in the setting of pancreatic trauma is both diagnostic (determination of AAST grade of pancreatic injury) and therapeutic (insertion of bridge prosthesis over contused region, sphincterotomy for reduction of intra-ductal pressure), but is associated with the need for repeated procedures for complications of stent placement, and ultimately for stent removal. $^{1,6}$

There is no gold standard currently with regards to the management of patients with pancreatic lacerations. A general guideline exists for conservative management of AAST class I and II pancreatic injuries and surgical management of AAST class III-V pancreatic injuries. ${ }^{2,5} \mathrm{~Pa}-$ tients with concomitant solid organ injuries, especially hepatobiliary or duodenal lacerations are most commonly treated surgically. ${ }^{4}$ Significant morbidity, mortality and protracted hospital stays have historically been reported in patients with AAST grade III-V pancreatic injuries being treated conservatively, with early surgical intervention proving more often than not to be the most expedious route to a full recovery in these patients. ${ }^{7-11}$ Conservative management includes a combination of expectant observation in a high dependency unit, fluid resuscitation, analgesia, endoscopic (ERCP) intervention, angioembolization (pancreatic injuries associated with intra-abdominal hemorrhage or lacerated blood vessels) and percutaneous drainage of peripancreatic collections and hematomas. The use of antibiotics or octreotide in these patients is controversial with no substantial supporting evidence. ${ }^{1}$ Surgical options include distal pancreatectomy with or without splenectomy, pancreaticoduodenectomy and non-anatomic pancreatic resections combined with repair or resection of other injured solid organs. A guiding principle has been described for the choice of surgical intervention - distal pancreatectomy with or without splenectomy for pancreatic ductal lesions to the left of the superior mesenteric vascular bundle; partial pancreatic resection and pancreatico-jejunostomy or a Whipple procedure for pancreatic ductal lesions to the right of the superior mesenteric vascular bundle. ${ }^{5}$ The operative management, especially pancreaticoduodenectomy, of pancreatic injuries has been strongly associated with a higher morbidity and mortality as compared to endoscopic or percutaneous procedures. ${ }^{1,2}$ This must, however, be considered with knowledge of the confounding severity of pancreatic injuries in patients who undergo surgical management, and the association of a higher AAST grade with more extensive intra-abdominal injuries. $^{1-5}$

The laparoscopic approach to management of traumatic pancreatic injuries has been poorly described in the literature. Published case reports on such attempts are few and far between, with the majority of cases being performed on isolated pancreatic injuries. ${ }^{6,9-11}$ Surgical technique for the laparoscopic approach was mostly similar amongst current case reports. All reports in current literature made use of 3 laparoscopic working ports with a single umbilical camera port, tacking sutures to retract the stomach anteriorly to the abdominal wall, division of gastrocolic ligament for access to the lesser sac, careful identification and dissection of splenic vessels apart from pancreatic tissue, and the use of surgical drains both for therapeutic and monitoring purposes post-operatively.

Rutkoski et. al. reported a unique method of intra-operative pancreatic transection with laparoscopic oversewing PDS 5-0 sutures to imbricate the transected surface and achieve closure. ${ }^{9}$ This was performed because the patient in question was an 8-year-old girl and weighed only 24 $\mathrm{kg}$. An endostapler was thus not used due to concerns of compromising more normal pancreatic tissue than necessary. This patient had no post-operative complications. This laparoscopic method of imbricating pancreatic tissues using laparoscopic suturing techniques instead of conventional stapling is reported also by Reynolds et al. In this case, the main pancreatic duct was sutured closed with 3-0 silk and the transected end of the pancreas closed with horizontal mattress sutures of 3-0 silk. Indication for this was similarly that of small pancreatic tissue found intra-operatively (patient was 18 -years-old). ${ }^{6}$ This patient similarly had no post-operative complications. Another unique technique reported by $\mathrm{Li}$ et. al. involved resection of the lacerated site between 2 endoscopic staplers instead of $1 .^{10}$ The acceptance of a remnant redundant pancreatic tail saved on the need for tedious and time-consuming intra-operative splenic hilar dissection. This patient had no 


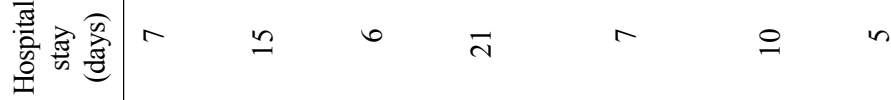

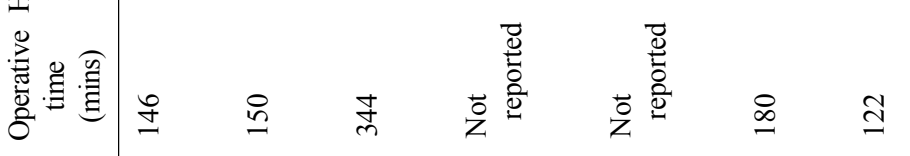

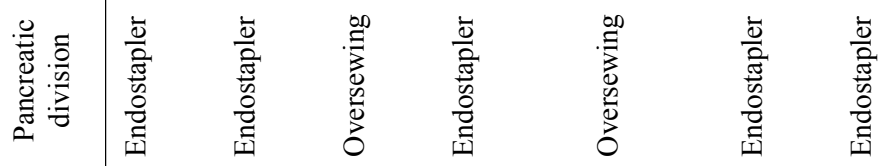

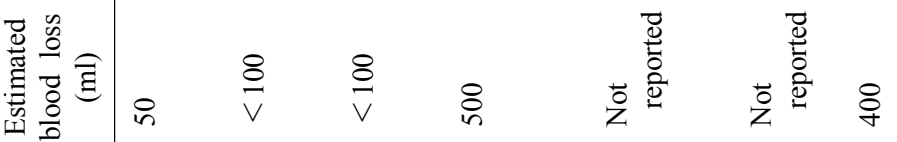

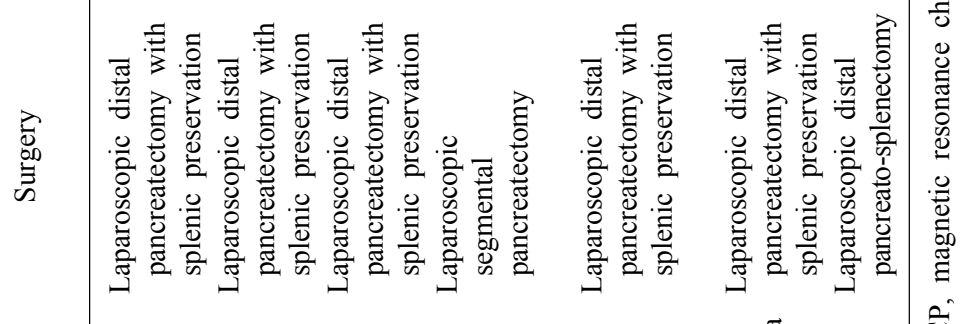

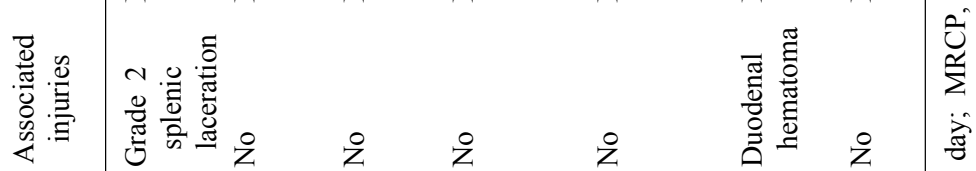

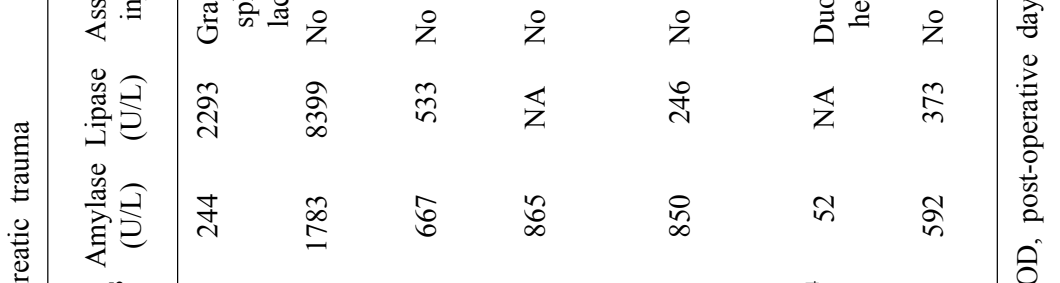

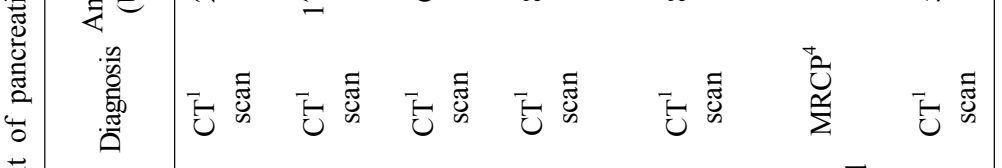

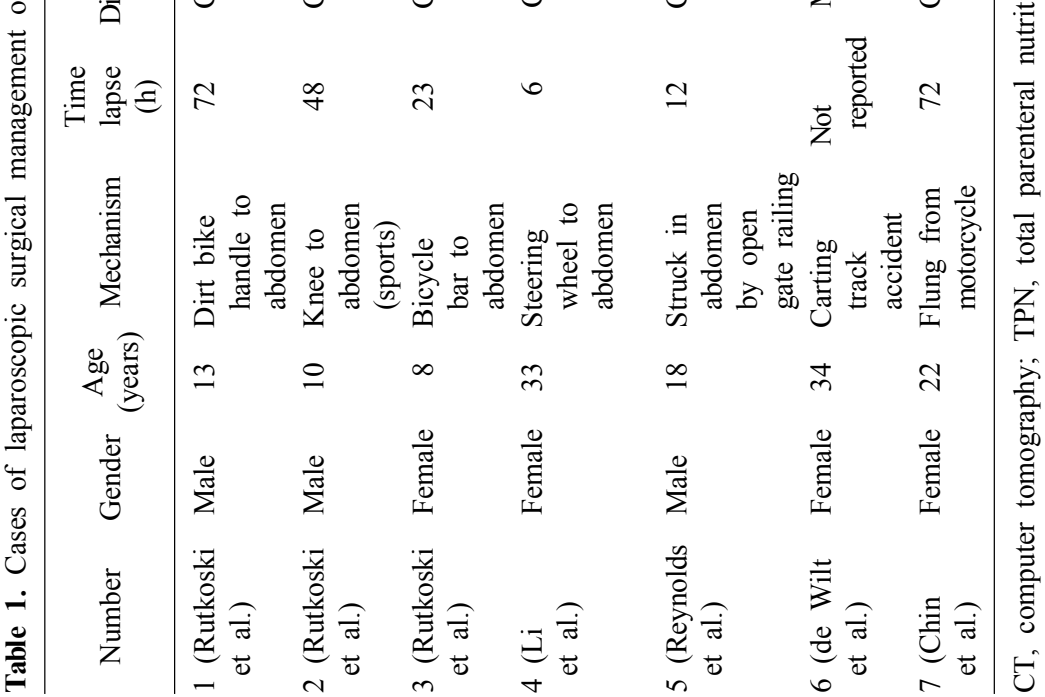


post-operative complications.

Complications after a laparoscopic pancreatectomy in the setting of trauma described in current literature include abdominal wall hematoma, prolonged ileus and peripancreatic collections. ${ }^{9,10}$ Even though length of stay after a laparoscopic approach is generally shorter than that after an open pancreatectomy, there have been insufficient case numbers to make any comparison statistically significant.

Our case report is unique in two aspects. We are one of few to describe the procedure of laparoscopic distal pancreatectomy combined with splenectomy performed for pancreatic trauma. The good outcome of this patient suggests that a combined pancreatosplenectomy is a safe and viable alternative in the setting of laparoscopic surgery in pancreatic trauma that ensures all surrounding vasculature undergoes definitive hemostasis and the surgical bed is kept as bloodless as possible. In addition, peripancreatic inflammation, edema and hematoma could make dissection and preservation of splenic vessels in the hilum tedious, time consuming and potentially unsuccessful. Hence, the operation time in the present case was shorter than previous reports whereby spleen preserving pancreatectomy was performed. ${ }^{9-11}$ It is important to highlight that pancreatosplenectomy was possible in the present case as our patient was an adult whereas many of the previous reported cases were in pediatric patients whereby preservation of the spleen is especially important. ${ }^{6,9}$

Besides this, our case report is one of few in the literature thus far where the patient was not subjected to total parenteral nutrition post-operatively. She was allowed feeds on POD2 and diet on POD3. Given our patient's smooth post-operative recovery, we postulate that early feeding is non-inferior to late feeding should pancreatic transection margins be adequately imbricated and hemostasis sufficiently secured. Table 1 summarizes all cases of laparoscopic surgical management of pancreatic trauma.

Finally, it is important to emphasize that LDP in the setting of trauma should only be performed by pancreatic surgeons experienced in laparoscopic surgery due to the increased complexity of these cases whereby the presence of a large hematoma and increased potential for bleeding frequently result in a distorted surgical anatomy with diminished visualization. In this case the primary surgeon had significant prior experience with major laparoscopic surgery such as minimally-invasive liver resections and pancreatectomies including pancreatoduodenectomies. ${ }^{12-14}$

Laparoscopic distal pancreatectomy is feasible for isolated blunt pancreatic trauma when performed by an experienced laparoscopic pancreatic surgeon. In such a setting, the laparoscopic approach can be considered as a viable alternative to open surgery, potentially offering the usual benefits of minimally invasive surgery. Further studies comparing between the open and laparoscopic approach are needed to determine if laparoscopic approach is superior to the open approach for blunt pancreatic trauma.

\section{ACKNOWLEDGEMENTS}

The corresponding author is not a recipient of a research scholarship. This paper is not based on any previous communication to any society or meeting. All work was performed at Singapore General Hospital, 1 Hospital Drive, Singapore 169608.

\section{REFERENCES}

1. Menahem B, Lim C, Lahat E, Salloum C, Osseis M, Lacaze L, et al. Conservative and surgical management of pancreatic trauma in adult patients. Hepatobiliary Surg Nutr 2016;5:470-477.

2. Machado MA, Volpe P, Souza Júnior AL, Poggetti RS, Branco PD, Birolini D. [Traumatic injuries of the pancreas: report of 65 cases]. Rev Hosp Clin Fac Med Sao Paulo 1994;49:238-242. Portuguese.

3. Vasquez JC, Coimbra R, Hoyt DB, Fortlage D. Management of penetrating pancreatic trauma: an 11-year experience of a level-1 trauma center. Injury 2001;32:753-759.

4. Ludwig K, Petermann J, Lorenz D. Diagnosis and therapy of traumatic injury of the pancreas. Zentralblatt Chir 1998;123:245250 .

5. Sukul K, Lont HE, Johannes EJ. Management of pancreatic injuries. Hepatogastroenterology 1992;39:447-450.

6. Reynolds EM, Curnow AJ. Laparoscopic distal pancreatectomy for traumatic pancreatic transection. J Pediatr Surg 2003;38:E7E9.

7. Nadler EP, Gardner M, Schall LC, Lynch JM, Ford HR. Management of blunt pancreatic injury in children. J Trauma 1999; 47:1098-1103.

8. Meier DE, Coln CD, Hicks BA, Guzzetta PC. Early operation in children with pancreas transection. J Pediatr Surg 2001;36: 341-344.

9. Rutkoski JD, Segura BJ, Kane TD. Experience with totally laparoscopic distal pancreatectomy with splenic preservation for pediatric trauma--2 techniques. J Pediatr Surg 2011;46:588-593.

10. Li JC, Ng SS, Teoh AY, Leung KL. Laparoscopic spleen-preserving pancreatectomy for traumatic pancreatic transection: a case report. Surg Laparosc Endosc Percutan Tech 2006;16:41-43.

11. de Wilt JH, van Eijck CH, Hussain SM, Bonjer HJ. Laparoscopic spleen preserving distal pancreatectomy after blunt abdominal trauma. Injury 2003;34:233-234.

12. Goh BKP, Lee SY, Teo JY, Kam JH, Jeyaraj PR, Cheow PC, 
et al. Changing trends and outcomes associated with the adoption of minimally invasive hepatectomy: a contemporary single-institution experience with 400 consecutive resections. Surg Endosc 2018;32:4658-4665.

13. Goh BKP, Lee SY, Kam JH, Soh HL, Cheow PC, Chow PKH, et al. Evolution of minimally invasive distal pancreatectomies at a single institution. J Minim Access Surg 2018;14:140-145. 14. Goh BKP, Low TY, Kam JH, Lee SY, Chan CY. Initial experience with laparoscopic and robotic surgery for the treatment of periampullary tumours: single institution experience with the first 30 consecutive cases. ANZ J Surg 2019;89:E137-E141. 